\title{
Negative Differential Conductivity in AIGaN/GaN HEMTs: Real Space Charge Transfer from 2D to 3D GaN States?
}

\author{
J. Deng, R. Gaska, M. S. Shur, M. A. Khan ${ }^{1}$, J. W. Yang ${ }^{1}$ \\ Department of ECSE and CIEEM, Rensselaer Polytechnic Institute \\ Troy, New York 12180, USA \\ ${ }^{1}$ Department of ECE, University of South Carolina \\ Columbia, South Carolina 29208, USA
}

\begin{abstract}
We report on non-thermal negative differential conductivity (NDC) in AlGaN/GaN HEMTs grown on sapphire substrates by low-pressure MOCVD. The sheet electron density was on the order of few times $10^{12} \mathrm{~cm}^{-2}$ and the Hall mobility was $1,000 \mathrm{~cm}^{2} / \mathrm{V}$.s. The HEMTs had threshold voltage close to zero and could operate at high positive gate bias up to 3 to 3.5 Volts, with a very low gate leakage current. NDC was observed at the gate bias larger than $1.5 \mathrm{~V}$ and at the drain biases between approximately $0.5 \mathrm{Vg}$ and $\mathrm{Vg}$. We excluded the possibility of self-heating as the cause, since the NDC occurs at relatively small power levels where self-heating effects are negligible.

An explanation we provided for the NDC effect is the new mechanism of real space charge transfer from 2D to 3D GaN states, which leads to a decrease in the channel mobility at large 2D electron gas densities. The observed low leakage can be explained by an enhanced molar fraction of aluminum at the heterointerface that results in a larger conduction band discontinuity. Our model that accounts for the piezoelectric and pyroelectric effects is consistent with the observed NDC effect. The Hall mobility dependence on the gate bias and sheet carrier concentration [1] is consistent with the real space transfer mechanism.

This NDC effect in GaN/AlGaN HEMTs may find applications in high-performance digital circuits at elevated temperatures.
\end{abstract}

\section{INTRODUCTION}

Recently, there has been much progress in the research and development of AlGaN/GaN High Electron Mobility Transistors (HEMTs). However, the physics of these novel devices has not yet been fully understood. In this paper, we report on a new mechanism of the negative differential mobility in $\mathrm{AlGaN} / \mathrm{GaN}$ HEMTs.

Negative differential conductance (NDC) in HEMTs was previously observed [2-3]. However, in these publications, the NDC was attributed to either gate leakage current [2] or self-heating effect [3]. In [2], it was claimed that the two dimensional electron gas was heated in the channel. This heating caused the "real-space transfer" [4] between the GaAs conducting layer and the AlGaAs barrier layer. Self-heating might also lead to NDC [3] since with the maximum sourcedrain current of $1 \mathrm{~A} / \mathrm{mm}$, the GaN devices grown on sapphire biased at $5 \mathrm{~V}$ could have a temperature rise of $125 \mathrm{~K}$, which would significantly reduce the mobility and the maximum charge density of the channel.

In our samples, these mechanisms do not play an important role for the following reasons. Self-heating is not important since the power dissipation in our samples is only on the order of $0.5 \mathrm{~W} / \mathrm{mm}$. For an estimated impedance of $25 \mathrm{~W} / \mathrm{mm}$ [3], this power dissipation should lead to the temperature rise of $12 \mathrm{~K}$, which is not enough to cause NDC. 
As shown below, piezoelectric and pyroelectric effects in $\mathrm{AlGaN} / \mathrm{GaN}$ heterostructures hinder the real space transfer into the AlGaN layer. However, as we discuss below, real space transfer into the $3 \mathrm{D}$ states in $\mathrm{GaN}$ is a possible mechanism explaining the observed NDC.

\section{EXPERIMENTS}

The $\mathrm{Al}_{0.2} \mathrm{Ga}_{0.8} \mathrm{~N} / \mathrm{GaN}$ HEMTs were grown on sapphire substrates by low-pressure metal organic chemical vapor deposition (MOCVD). The epilayer design of the samples was described in [5]. The measured 2DEG sheet density was on the order of $10^{12} \mathrm{~cm}^{-2}$ and the Hall mobility was $1,000 \mathrm{~cm}^{2} /$ V.s.

Figure 1(a) shows the current-voltage characteristics of the $45 \mu \mathrm{m}$ wide AlGaN-GaN HEMT. The source-drain spacing $\mathrm{L}_{\mathrm{ds}}$ was $5 \mu \mathrm{m}$ and the gate length $\mathrm{L}_{\mathrm{g}}$ was $1.7 \mu \mathrm{m}$. The applied gate bias was as high as $3.0 \mathrm{~V}$. The negative differential conductivity (NDC) was observed at the gate biases $\mathrm{V}_{\mathrm{g}}>1.5 \mathrm{~V}$. The drain current increased with $\mathrm{V}_{\mathrm{ds}}$ to the peak value, $\mathrm{I}_{\text {peak }}$, determined by the gate bias, and then decreased until saturation current $\left(\mathrm{I}_{\mathrm{sat}}\right)$ has been reached. For HEMTs with the drainto-gate spacing of $1 \mu \mathrm{m}$ and $2 \mu \mathrm{m}$ the peak drain current was achieved at the source-drain voltages of approximately $\mathrm{V}_{\text {peak }}=1.5 \mathrm{~V}$ and $\mathrm{V}_{\text {peak }}=2.5 \mathrm{~V}$, respectively. The NDC was observed at the source-drain voltages $\mathrm{V}_{\text {peak }}<\mathrm{V}_{\mathrm{ds}}<\mathrm{V}_{\text {valley, }}$, where $\mathrm{V}_{\text {valley }}$ was the drain bias when the NDC effect stopped. The drain current reached its saturation beyond $V_{\text {valley. }}$. In Figure 1(b), we show the gate bias dependence of $V_{\text {peak }}, V_{\text {valley }}$ and the peak-valley current ration $R=I_{\text {peak }} / I_{\text {valley }}$. This figure demonstrates that $\mathrm{V}_{\text {valley }}$ increased with the gate bias with a slope of about 1 , while the increase rate of Vpeak was much smaller. On the other hand, $\mathrm{R}$ also increased almost linearly with $\mathrm{V}_{\mathrm{g}}$.

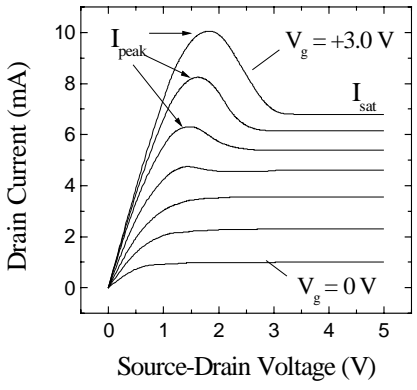

(a)

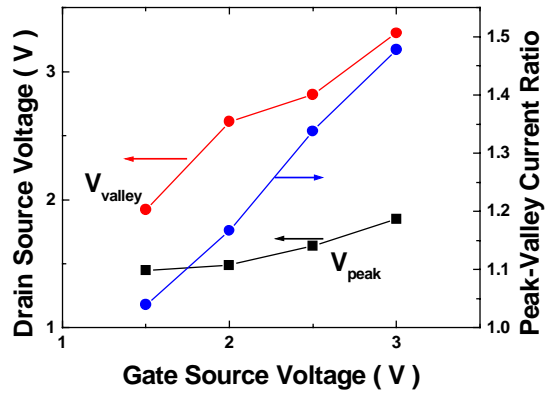

(b)

Figure 1. Drain current-voltage characteristics of the $45 \mu \mathrm{m}$ wide AlGaN-GaN HEMT. (a) Drain current dependence on $\mathrm{Vd}, \mathrm{Vg}$ varies from 0 to $3 \mathrm{~V}$ with the step of $0.5 \mathrm{~V}$. (b) The $\mathrm{V}_{\text {peak }}, \mathrm{V}_{\text {valley }}$ and $\mathrm{I}_{\text {peak }} / \mathrm{I}_{\text {sat }}$ as functions of the gate bias.

We measured NDC in the HEMTs with the source-drain spacing from $5 \mu \mathrm{m}$ to $15 \mu \mathrm{m}$ and the offset gate length from $2 \mu \mathrm{m}$ to $12 \mu \mathrm{m}$. The source-to-gate and gate-to-drain distances were approximately the same and equal to $2 \mu \mathrm{m}$ and $1 \mu \mathrm{m}$, respectively. Figure 2 demonstrates the normalized current drop as a function of the gate length for $95 \mu \mathrm{m}$ wide HEMTs $\left(\Delta \mathrm{I}_{\text {neg }}=\mathrm{I}_{\text {peak }}-\mathrm{I}_{\text {sat }}\right)$. The obtained results clearly showed strong dependence of NDC on $\mathrm{L}_{\mathrm{g}}$. At the gate bias of $+3.0 \mathrm{~V}$, the increase in the gate length for about 6 times (source-drain spacing increases 3 times) reduces NDC by a factor of larger than 30 . The saturation current for the same gate lengths decreases 
linearly only by one third, from $12 \mathrm{~mA}$ to $8 \mathrm{~mA}$. The $\Delta \mathrm{I}_{\text {neg }}$ dependence on the gate length can be described by expression $\Delta \mathrm{I}_{\text {neg }} \sim \mathrm{L}_{\mathrm{g}}^{-\alpha}$, where $\alpha$ is from 1.5 to 2 .

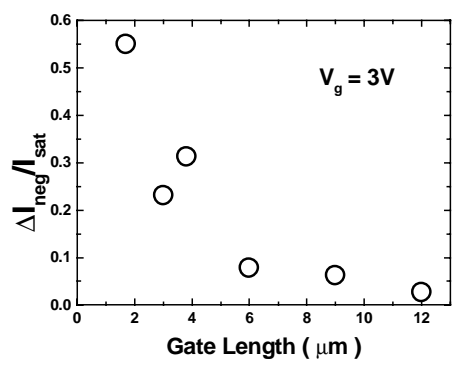

Figure 2. Gate length dependence of the current drop in NDC (normalized to the $\mathrm{I}_{\text {sat }}$ )

\section{DISCUSSION}

The gate current versus drain voltage at different gate biases for the device in Figure 1 was measured. (See also [6]). Even for the zero source-drain voltage, the gate leakage current at high positive gate bias $\left(\mathrm{V}_{\mathrm{g}}=+3.0 \mathrm{~V}\right)$ was less than $0.5 \mathrm{~mA}$, whereas the drain current reduction in the NDC region for the same gate bias was more than $3 \mathrm{~mA}$ (see Figure 1(a)). At the source-drain voltage of $1.5 \mathrm{~V}$ and higher (NDC region) the gate current dropped below $1 \mu \mathrm{A}$. These results indicated that the gate current of the device were negligible compared to $\Delta \mathrm{I}_{\text {neg }}$.

As we mentioned before, the device had a maximum drain current on the order of $0.1 \sim 0.2 \mathrm{~A} / \mathrm{mm}$, which would only raise the temperature by $10 \sim 20 \mathrm{~K}$ at a thermal impedance of 25 $\mathrm{K} \cdot \mathrm{mm} / \mathrm{W}$ [3]. Also, the self-heating NDC led to a monotonous decrease of the drain current in the saturation regime, whereas the NDC reported here took place in a limited drain voltage range (see Figure 1(a)). Therefore, we concluded that both the gate current and self-heating were not the mechanism leading to the NDC in our samples.

The explanation we provided for the NDC was the mobility and sheet carrier density decrease due to the real space transfer from the $2 \mathrm{D}$ to $3 \mathrm{D} \mathrm{GaN}$ states. First, we compared the mobility of the two-dimensional and the three-dimensional electron gas. The results are shown in Figure 3. Even at room temperature, the mobility of 2D-electron gas is around 50\% larger than the mobility of 3D electrons. This difference is much larger at cryogenic temperatures.

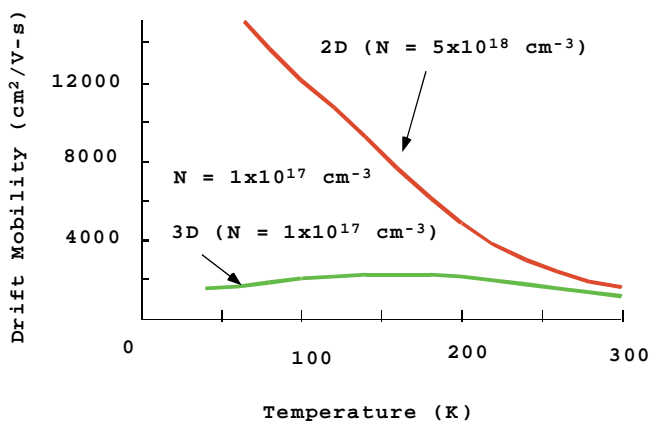

Figure 3. Calculated 2D and 3D electron mobility in $\mathrm{GaN}$ at different temperatures 
In Figure 4, we compared the band diagram of HEMTs in different material systems - GaNbased and GaAs-based. Due to the piezoelectric and pyroelectric effects, the conduction band profile in the AlGaN layer does not have a minimum that is needed for the real space transfer from $\mathrm{GaN}$ into AlGaN. Also, in contrast to $\mathrm{AlGaAs} / \mathrm{GaAs}$ system, the energy difference between the Fermi level in the 2D gas and the bottom of the conduction band in the $\mathrm{GaN}$ is relatively small due to the residual doping in GaN. This small difference made the electron transition into the GaN bulk more important.

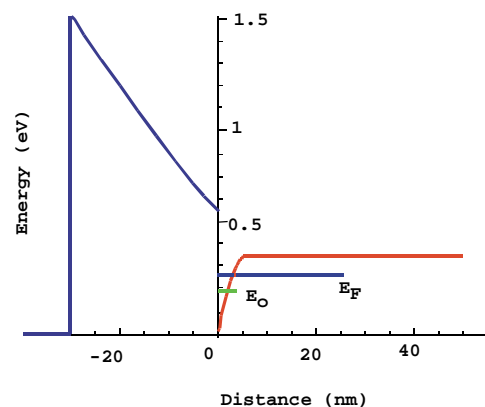

(a)

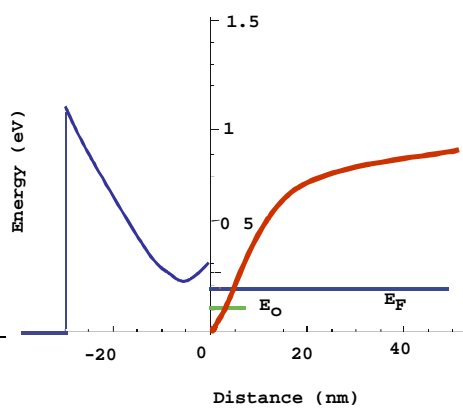

(b)

Figure 4. Comparison between the band diagram of GaN based HEMTs and GaAs based HEMTs. (a) Calculated band diagram of GaN HEMTs at the doping level of $10^{17} \mathrm{~cm}^{-3}$ (b) Qualitative band diagram of GaAs HEMTs

Hence, we believe that this transfer of 2D electrons into 3D GaN states reduces the channel mobility and the 2D-electron sheet concentration and leads to the current drop in the NDC region. The Monte-Carlo simulation results for AlGaAs/GaAs HEMTs reported in [7] also pointed out to the possibility of such a mechanism (see Figure 5). The dash-dotted line in Figure 5 shows the electron concentration in the quantum well and the full lines show the bulk GaAs electron concentration. This figure demonstrates that the electrons inside the quantum well experience the transition into the GaAs buffer along the channel. However, due to the low barrier height of the GaAs transistors (Figure 4(b)), the gate current becomes dominant at higher gate biases, which makes it difficult to observe the NDC related to such a transfer in GaAs devices at room temperature. 


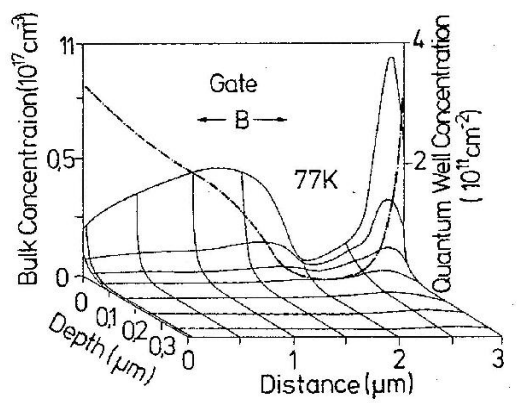

Figure 5. Monte Carlo simulation results on electron concentration in GaAs based HEMTs. Dashed dotted line, concentrations in a quantum well; full line, concentrations in bulk GaAs. After [7]

In order to illustrate this new mechanism of NDC, we calculated the current-voltage characteristics of our devices for different values of the electron mobility using the AIM-Spice [8], which helped to understand the device operation. In Figure 6(a), a mobility of $750 \mathrm{~cm}^{2} / \mathrm{V}$.s was used and reasonable agreement was reached in the low drain bias region. The mobility dropped to $400 \mathrm{~cm}^{2} /$ V.s in Figure 6(b), which give reasonable fit in the saturation region. The maximum sheet charge density for these two regions was also different. (See Table 1) These simulation results confirm that a mobility drop at the higher gate bias might be the cause of the NDC effect, as illustrated in Figure 3.

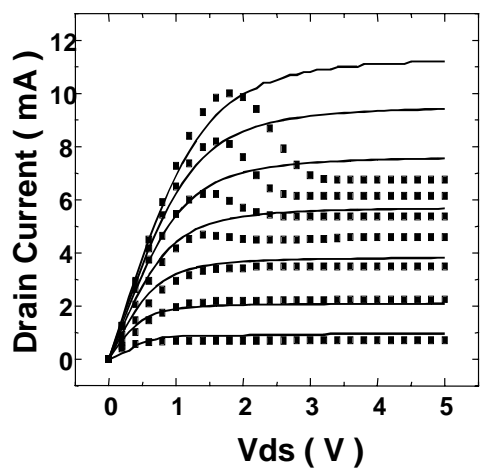

(a)

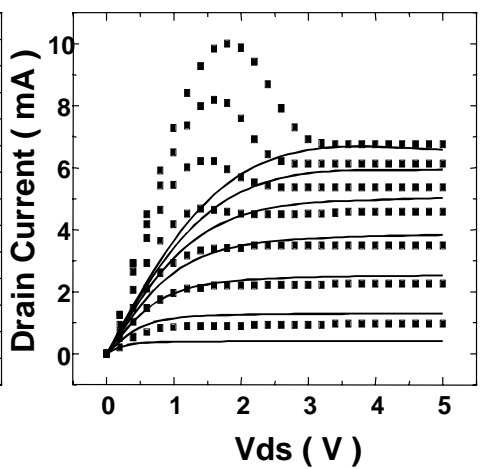

(b)

Figure 6. AIM-Spice model of the above device. The model parameters are in Table 1. The symbols are the experimental results and the lines are from simulation.

\section{CONCLUSIONS}

In this paper, we report the non-thermal negative differential conductance in $\mathrm{AlGaN} / \mathrm{GaN}$ HEMTs. With the help of device characterization and modeling, we excluded the possibility of selfheating and real space transfer into the AlGaN layer current as the causes of the NDC. We linked 
the NDC to the mobility reduction caused by the transfer from 2D to 3D states in the channel. This mechanism was supported by the device simulation and band diagram calculations.

\section{ACKNOWLEDGEMENTS}

The work has been supported by the Office of Naval Research (Project Monitor Dr. Colin Wood and Dr. John Zolper). We are also grateful to Dr. Knap and Dr. Roumiantsev for their useful discussions.

Table 1. AIM-Spice parameters for Figure 6.

\begin{tabular}{|l|l|l|l|}
\hline Parameters & $\begin{array}{l}2 \mathrm{D} \\
\text { channel }\end{array}$ & $\begin{array}{l}3 \mathrm{D} \\
\text { channel }\end{array}$ & Unit \\
\hline $\mathrm{W}_{\mathrm{g}}$ (Gate width) & 45 & 45 & $\mu \mathrm{m}$ \\
\hline $\mathrm{L}_{\mathrm{g}}$ (Gate length) & 1.7 & 1.7 & $\mu \mathrm{m}$ \\
\hline $\mathrm{D}_{\mathrm{i}}$ (Distance to buffer layer charge) & 30 & 30 & $\mathrm{~nm}$ \\
\hline$\lambda$ (Output conductance parameter) & 0 & 0 & $\mathrm{~V}^{-1}$ \\
\hline$\mu$ (Low-field mobility) & 0.075 & 0.04 & $\mathrm{~m}^{-2} \mathrm{~V}^{-1} \mathrm{~s}^{-1}$ \\
\hline $\mathrm{n}_{\max }$ (Sheet charge density) & $1 \bullet 10^{17}$ & $4 \bullet 10^{16}$ & $\mathrm{~m}^{-2}$ \\
\hline$\Phi_{\mathrm{b}}$ (Heterojunction barrier height) & 1.1 & 1.1 & $\mathrm{~V}$ \\
\hline $\mathrm{R}_{\mathrm{s}}$ (Drain series resistance) & 30 & 30 & $\Omega$ \\
\hline $\mathrm{R}_{\mathrm{d}}$ (Source series resistance) & 30 & 30 & $\Omega$ \\
\hline $\mathrm{V}_{\mathrm{s}}$ (Saturation velocity) & 40000 & 40000 & $\mathrm{~m} / \mathrm{s}$ \\
\hline $\mathrm{M}$ (Knee voltage parameter) & 2 & 2 & - \\
\hline $\mathrm{V}_{\mathrm{T}}$ (Zero-bias threshold voltage) & -0.6 & -0.6 & $\mathrm{~V}$ \\
\hline
\end{tabular}

\section{REFERENCES}

1. R. Gaska, M. S. Shur, A. D. Bykhovski, A. O. Orlov, and G. L. Snider, Appl. Phys. Lett. 74, 287 (1999)

2. M. S. Shur, D. K. Arch, R. R. Daniels, J. K. Abrokwah, IEEE Electron Device Lett. 7, 78 (1986)

3. R. Gaska, A. Osinsky, J. W. Yang and M. S. Shur, IEEE Electron Device Lett. 19, 89 (1998).

4. K. Hess, H. Markoc, H. Shichijo and B. G. Streetman, Appl. Phys. Lett. 35(b), 469 (1979)

5. R. Gaska, Q. Chen, M. Asif Khan, A. Ping, I. Adesida and M. S. Shur, Electron Lett. 33, 1255 (1997)

6. J. Deng, R. Gaska, M. S. Shur, M. A. Khan, J. W. Yang, submitted to Appl. Phys. Lett.

7. D. Widiger, K. Hess, J. J. Coleman, IEEE Electron Device Lett. 5, 266 (1984)

8. T. A Fjeldly, T. Ytterdal and M. Shur, Introduction to Device Modeling and Circuit Simulation (1997) 